\title{
BMJ Open Protocol of DREAM3R: DuRvalumab with chEmotherapy as first-line treAtment in advanced pleural Mesothelioma - a phase 3 randomised trial
}

\author{
Peey Sei Kok (D , ,,2 Patrick M Forde, ${ }^{3}$ Brett Hughes, ${ }^{4,5}$ Zhuoxin Sun, ${ }^{6}$ \\ Chris Brown, ${ }^{1}$ Suresh Ramalingam, ${ }^{7}$ Alistair Cook, ${ }^{8,9}$ Willem Joost Lesterhuis, ${ }^{10}$ \\ Sonia Yip, ${ }^{1}$ Ken O’Byrne, ${ }^{11,12}$ Nick Pavlakis, ${ }^{13,14}$ Julie Brahmer, ${ }^{3}$ \\ Valsamo Anagnostou, ${ }^{3}$ Kate Ford, ${ }^{1}$ Karen Fitzpatrick, ${ }^{15}$ Alison Bricker, ${ }^{15}$ \\ Michelle M Cummins, ${ }^{1}$ Martin Stockler, ${ }^{1}$ Anna K Nowak, ${ }^{8,16}$ Thoracic Oncology \\ Group of Australasia (TOGA) and PrECOG, USA
}

To cite: Kok PS, Forde PM, Hughes $B$, et al. Protocol of DREAM3R: DuRvalumab with chEmotherapy as first-line treAtment in advanced pleural Mesothelioma-a phase 3 randomised trial. BMJ Open 2022;12:e057663. doi:10.1136/ bmjopen-2021-057663

- Prepublication history for this paper is available online. To view these files, please visit the journal online (http://dx.doi. org/10.1136/bmjopen-2021057663).

Received 27 September 2021 Accepted 16 December 2021

Check for updates

(c) Author(s) (or their employer(s)) 2022. Re-use permitted under CC BY-NC. No commercial re-use. See rights and permissions. Published by BMJ.

For numbered affiliations see end of article.

Correspondence to

Dr Peey Sei Kok;

peey-sei.kok@sydney.edu.au

\section{ABSTRACT}

Introduction There is a strong theoretical rationale for combining checkpoint blockade with cytotoxic chemotherapy in pleural mesothelioma and other cancers. Two recent single-arm, phase 2 trials [DuRvalumab with chEmotherapy as first-line treAtment in advanced pleural Mesothelioma (DREAM) and Phase II multicenter study of anti-PD-L1, durvalumab, in combination with cisplatin and pemetrexed for the first-line treatment of unresectable malignant pleural mesothelioma (PrE0505)] combining the programmed death ligand-1 (PD-L1) inhibitor durvalumab with standard first-line chemotherapy exceeded prespecified safety and activity criteria to proceed to a phase 3 confirmatory trial to assess this combination. We present the protocol of the DREAM3R trial.

Methods and analysis This multicentre open-label randomised trial will recruit 480 treatment-naïve adults with advanced pleural mesothelioma, randomised $(2: 1)$ to either 3-weekly durvalumab $1500 \mathrm{mg}$ plus 3-weekly doublet chemotherapy (cisplatin $75 \mathrm{mg} / \mathrm{m}^{2}$ or carboplatin, Area Under the Curve,AUC 5 and pemetrexed $500 \mathrm{mg} / \mathrm{m}^{2}$ ) 4-6 cycles, followed by 4-weekly durvalumab $1500 \mathrm{mg}$ until disease progression, unacceptable toxicity or patient withdrawal; OR doublet chemotherapy alone for 4-6 cycles, followed by observation. The target accrual time is 27 months, with follow-up for an additional 24 months. This provides over $85 \%$ power if the true HR for overall survival (OS) is 0.70 , with two-sided alpha of 0.05 , assuming a median $0 S$ of 15 months in the control group. Randomisation is stratified by age (18-70 years vs $>70$ ), sex, histology (epithelioid vs non-epithelioid), platinum agent (cisplatin vs carboplatin) and region (USA vs Australia/New Zealand vs Other). The primary endpoint is OS. Secondary endpoints include progression-free survival, objective tumour response (by mRECIST V.1.1 and iRECIST), adverse events, health-related quality of life and healthcare resource use. Tertiary correlative objectives are to explore and validate potential prognostic and/or predictive biomarkers (including features identified in the DuRvalumab with chEmotherapy as first-line treAtment in advanced pleural Mesothelioma (DREAM) and PrE0505

\section{Strengths and limitations of this study}

- International, open-labelled, randomised phase 3 trial of immunotherapy and chemotherapy in firstline treatment of pleural mesothelioma.

- Strong biological rationale and earlier phase clinical data.

- Extensive translational science biospecimen collection and plans.

- This study does not contain a comparator arm of ipilimumab-nivolumab combination, which is an option for first-line treatment, particularly for sarcomatoid disease

- The control arm (cisplatin/carboplatin plus pemetrexed) does not include bevacizumab, which is an option for first line treatment.

studies, PD-L1 expression, tumour mutational burden, genomic characteristics and human leukocyte antigen subtypes) in tissue and serial blood samples. An imaging databank will be assembled for validation of radiological measures of response, and studies of possible radiomic biomarkers in mesothelioma.

Ethics and dissemination The protocol was approved by human research ethics review committees for all participating sites. Results will be disseminated in peerreviewed journals and at scientific conferences.

Drug Supply AstraZeneca.

Protocol version CTC 0231 / TOGA 18/001 / PrE0506 $3.0,29$ July 2021.

Trial registration number ClinicalTrials.gov Identifier: NCT04334759 ACTRN 12620001199909.

\section{INTRODUCTION}

The incidence of pleural mesothelioma continues to rise worldwide, particularly in Asia, despite bans on using asbestos in many countries. ${ }^{1}$ The annual incidence rates in the UK, Australia and the USA in 2019-2021 were 
6.8, 4.2 and 1.51 per 100000 people, respectively. ${ }^{2-4}$ Once diagnosed, mesothelioma is generally incurable and has a median survival of less than 1 year. Systemic treatment with palliative intent is the only option for the majority of patients. ${ }^{5-7}$ Hundreds of thousands of people worldwide will require systemic therapy for mesothelioma in coming decades.

\section{Current treatment options in mesothelioma}

Since 2003, the standard first-line chemotherapy for advanced unresectable pleural mesothelioma has been platinum-based chemotherapy with 4-6 cycles of cisplatin or carboplatin, with Pemetrexed. This provides a median survival benefit of approximately 3 months over cisplatin alone in the pivotal trail and benefits in patient-reported outcomes. ${ }^{5-8}$ More recently, the combination of chemotherapy with bevacizumab, a vascular endothelial growth factor inhibitor, demonstrated an additional median survival benefit of 2 months. ${ }^{9}$ However, this combination was not approved by the US FDA and most other regulatory bodies. Addition of the multitargeted tyrosine kinase inhibitor nintedanib to chemotherapy also resulted in a progressionfree survival (PFS) benefit in a randomised phase 2 trial, but a subsequent randomised phase 3 trial was negative. ${ }^{10}$ The majority of patients who respond to first-line therapy experience tumour progression soon after it is completed. Thus, there has been a strong unmet clinical need to improve firstline systemic therapy in mesothelioma.

\section{Rationale for immunotherapy in mesothelioma}

The activity of immunotherapy in mesothelioma has been demonstrated in second and subsequent line studies. ${ }^{11-18}$ In earlier studies, outcomes with single agent immunotherapy varied with the population and setting, but clearly indicated activity. The recent randomised phase 3 PROMISE trial showed similar outcomes with single agent pembrolizumab versus single agent chemotherapy when used as second-line treatment. ${ }^{17}$ However, nivolumab provided benefits in progression-free and overall survival (OS) in comparison with best supportive care in the second-line or subsequent-line setting. Trials of dual immunotherapy as second-line treatment have shown longer PFS than single agent immunotherapy in the Bevacizumab for newly diagnosed pleural mesothelioma in the Mesothelioma Avastin Cisplatin Pemetrexed Study (MAPS-2) ${ }^{14}$ and Tremelimumab plus durvalumab retreatment and 4-year outcomes in patients with mesothelioma: a follow-up of the open label, non-randomised, phase 2 NIBIT-MESO- ${ }^{19}$ studies; however, these observations require further validation in larger trials with OS as the primary end point to outweigh the toxicities of dual immunotherapy.

The recently reported results of the CheckMate-743 phase 3 randomised trial of dual immunotherapy with ipilimumab and nivolumab as first-line chemotherapy showed a significant improvement in OS when compared with a platinum-based doublet (HR 0.74; 95\% CI 0.60 to 0.91; $\mathrm{p}=0.002) .{ }^{20}$ However, the OS benefit was observed predominantly in the subgroup with non-epithelioid histology (25\% of participants), rather than among the more common subgroup with epithelioid histology (75\% of participants). There was also no benefit observed in PFS or objective response rate (ORR) compared with chemotherapy.

\section{First-line combination of immunotherapy and chemotherapy}

Durvalumab is a human monoclonal antibody of the IgG) 1 kappa subclass that inhibits binding of PD-L1. The proposed mechanism of action for durvalumab is interference in the interaction of PD-L1 with PD1 and CD80 (B7.1). In vivo studies have shown that durvalumab inhibits tumour growth in xenograft models via a T-cell-dependent mechanism. ${ }^{21}$ Based on these data, durvalumab is expected to stimulate the patient's antitumor immune response by binding to PD-L1 and shifting the balance towards an antitumor response.

Risks with durvalumab include, but are not limited to, diarrhoea/colitis, pneumonitis/interstitial lung disease (ILD), endocrinopathies (ie, events of hypophysitis/hypopituitarism, adrenal insufficiency, hyperthyroidism and hypothyroidism, type I diabetes mellitus and diabetes insipidus), hepatitis/increases in transaminases, nephritis/increases in creatinine, rash/dermatitis including pemphigoid, myocarditis, myositis/polymyositis, immune thrombocytopenia, infusion-related reactions, hypersensitivity reactions, pancreatitis, serious infections and other rare or less frequent inflammatory events including neuromuscular toxicities (eg, GuillainBarré syndrome, myasthenia gravis).

Two recent single-arm first-line phase 2 trials $^{22} 23$ combining durvalumab with platinum-based doublet chemotherapy showed encouraging activity and acceptable safety in advanced mesothelioma of all subtypes. The Australian DREAM trial of 54 participants exceeded its prespecified target with a 6-month PFS rate of $57 \% .^{22}$ The ORR was $48 \%$, median PFS was 7 months by mRECIST and iRECIST; median OS was 18 months. In the US-based PrECOG 0505 trial of 55 participants, the median OS was 20.4 months, 12-month OS rate was $70 \%$ (95\% CI 56 to 81 ), ORR was $56 \%$, 6-month PFS rate was $69.1 \%$ and median PFS was 7 months. ${ }^{23}$

DREAM3R was designed and developed before results from CheckMate-743 were available. The positive results of CheckMate-743 strengthen the strong rationale for DREAM3R. The OS benefit in Checkmate-743 was uncertain in the subgroup with epithelioid histology, and not evident in the subgroup with tumours that did not express PD-L1. Results of translational research studies to identify those more likely to benefit are pending.

Given the favourable OS and PFS data from the DREAM and PrE0505 trials, DREAM3R aims to determine the effectiveness of adding durvalumab to cisplatin/carboplatin and pemetrexed. Our primary hypothesis is that the addition of durvalumab will prolong OS in comparison with platinum and pemetrexed alone. 


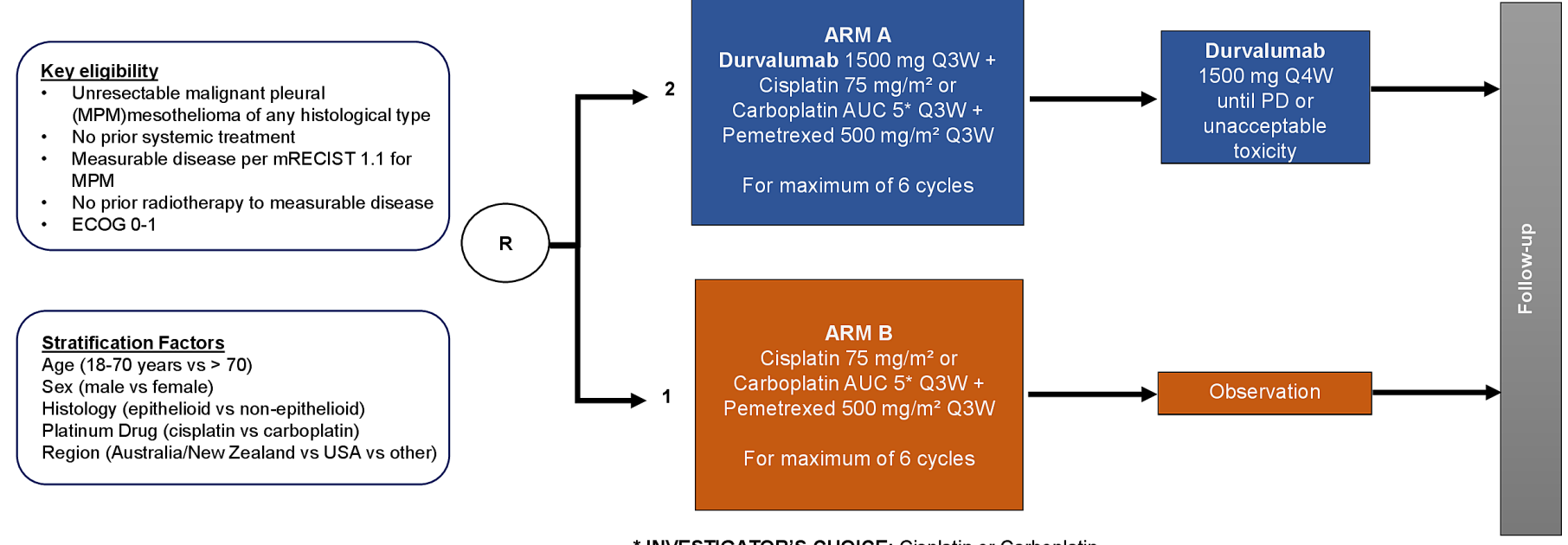

* INVESTIGATOR'S CHOICE: Cisplatin or Carboplatin

Primary endpoint: OS
Secondary endpoints: PFS, OTRR, AEs, HRQoL, healthcare resources
Tertiary endpoints: Possible prognostic/predictive biomarkers in tissue and serial blood samples: PD-L1, HLA subtypes, tumour mutation burden, genomic characteristics;
validation of radiological measures of response and radiomic biomarkers

Figure 1 Schema for DREAM3R. AEs, adverse events, DREAM3R, DuRvalumab with chEmotherapy as first-line treAtment in advanced pleural Mesothelioma-phase 3 randomised trial; ECOG, Eastern Cooperative Oncology Group; HRQoL, healthrelated quality of life, HLA, human leukocyte antigen; OS, overall survival; OTRR, objective tumour response rate; PD-L1, programmed death ligand-1; PFS, progression-free survival.

\section{METHODS AND ANALYSES \\ Trial design}

The DREAM3R trial is an international, open label, randomised (2:1), multicentre, phase 3 trial. The planned study sites include 29 in Australia, 1 in New Zealand and 30 from the USA.

Participants are randomised in a ratio of 2:1 to either durvalumab + chemotherapy or chemotherapy alone, by a central computerised system that uses permuted blocks to stratify for (see figure 1):

1. Age (18-70 years vs older than 70).

2. Sex (male vs female).

3. Histology (epithelioid vs non-epithelioid).

4. Region (Australia/New Zealand vs USA vs other).

5. Platinum agent (cisplatin vs carboplatin).

Eastern Cooperative Oncology Group (ECOG) is not a stratification factor. It is unlikely that there will be equal numbers of ECOG 0 and ECOG 1 recruited.

\section{Inclusion criteria}

Participants who fulfil these criteria are considered eligible:

- Adults with a histological diagnosis of pleural mesothelioma of any histological type, that is, not amenable to curative surgical resection. Histological diagnosis requires tumour tissue from an open biopsy or a core biopsy with a needle of 19 gauge or wider.

- Measurable disease per mRECIST V.1.1 for pleural mesothelioma.

- No prior radiotherapy to measurable disease.

- ECOG score 0 to 1.

- Tumour tissue- formalin-fixed paraffin-embedded (FFPE) available from diagnostic biopsy for PD-L1.
- Adequate blood tests (done within 14 days prior to randomisation) and with values within the ranges specified below. Blood transfusions are permissible if completed at least 7 days prior to treatment start.

- Haemoglobin $\geq 9.0 \mathrm{~g} / \mathrm{L}$.

- Absolute neutrophil count $\geq 1.5 \times 10^{9} / \mathrm{L}$.

- Platelets $\geq 100 \times 10^{9} / \mathrm{L}$.

- Total bilirubin $\leq 1.5 \times$ upper limit of normal (ULN) (except participants with Gilbert's syndrome, who are eligible with bilirubin $\leq 2.5 \mathrm{ULN}$ ).

- Alanine transaminase $\leq 2.5 \times$ ULN, unless liver metastases or invasion are present, in which case, it must be $\leq 5 \times$ ULN.

- Aspartate aminotransferase $\leq 2.5 \times$ ULN, unless liver metastases or invasion are present, in which case, it must be $\leq 5 \times$ ULN.

- Creatinine clearance $(\mathrm{CrCl}) \geq 45 \mathrm{~mL} / \mathrm{min}$ (per Cockcroft-Gault formula).

- Life expectancy at least 12 weeks.

- Women of childbearing potential must use a reliable means of contraception during treatment and for at least 90 days thereafter. Breastfeeding is not permissible during or for at least 90 days after the final study treatment. Men must have been surgically sterilised or use a (double if required) barrier method of contraception if they are sexually active with a woman of childbearing potential.

- Evidence of postmenopausal status or negative serum pregnancy test for female premenopausal participants. Women will be considered postmenopausal if they have been amenorrheic for 12 months without an alternative medical cause. 


\section{Exclusion criteria}

Participants who meet the following criteria are considered ineligible

- Received prior chemotherapy, immune checkpoint inhibitor or other systemic anticancer therapy for pleural mesothelioma.

- Diagnosis on cytology or fine-needle aspiration only.

- Contraindication for immune checkpoint inhibitor such as active or documented autoimmune or inflammatory disorder.

- Any condition requiring systemic treatment with corticosteroids $(>10 \mathrm{mg} /$ day prednisone or equivalent) or other immunosuppressive medications within 28 days.

- Symptomatic or uncontrolled brain or leptomeningeal metastases.

- Hearing loss or peripheral neuropathy considered by the investigators to contraindicate cisplatin administration.

- History of allergy or hypersensitivity to investigational product, cisplatin, pemetrexed or any excipient.

- No other malignancy that requires active treatment. Participants with a previous history of adequately treated carcinoma in situ, non-melanoma skin cancer or lentigo maligna without evidence of disease or superficial transitional cell carcinoma of the bladder are eligible.

- Current treatment or treatment within the last 12 months with any investigational anticancer products.

- Concurrent enrolment in another clinical trial testing an anticancer treatment.

- Uncontrolled intercurrent illness including, but not limited to, ongoing or active infection, symptomatic congestive cardiac failure, uncontrolled hypertension, unstable angina pectoris, cardiac arrhythmia, ILD, active peptic ulcer disease or gastritis, serious chronic gastrointestinal conditions associated with diarrhoea, active bleeding diatheses.

- Hepatitis B, hepatitis C or HIV. Exceptions include past or resolved Hepatitis B (defined as the presence of hepatitis B core antibody and absence of hepatitis B surface antigen, HBsAg) and participants positive for hepatitis C (HCV) antibody if PCR is negative for HCV RNA. HIV testing is not required in absence of clinical suspicion of HIV.

- Known history of primary immunodeficiency, allogeneic organ transplant, pneumonitis or active tuberculosis.

- Receipt of live-attenuated vaccination within 30 days prior to enrolment or within 30 days of receiving durvalumab.

\section{Study objectives}

The primary objective of DREAM3R trial is to determine the effects of adding durvalumab on OS. Secondary objectives are to determine effects on

PFS (by mRECIST V.1.1 for pleural mesothelioma and iRECIST).
Objective tumour response ((OTR) by mRECIST V.1.1 for pleural mesothelioma and iRECIST).

Adverse events according to Common Terminology Criteria (CTCAE V.5.0).

Health-related quality of life (HRQL, EORTC QLQC30, QLQ LC-29, EQ-5D-5L).

Healthcare resource use.

Incremental cost-effectiveness.

Tertiary/correlative objectives are

To explore and validate potential prognostic or predictive biomarkers of clinical outcomes (including but not limited to candidates identified in the phase II DREAM and PrE0505 studies, PD-L1 expression, human leukocyte antigen type, $\mathrm{T}$ cell tumour infiltration, $\mathrm{T}$ cell receptor repertoire, tumour mutational burden and gene signatures).

To collect an imaging databank for future validation of radiological response metrics in pleural mesothelioma

\section{Treatment arms}

Investigational arm (arm A)

Standard doublet chemotherapy+durvalumab, followed by durvalumab maintenance (see table 1 ).

Cisplatin/carboplatin and pemetrexed are administered before durvalumab. Durvalumab is administered immediately following or during the final hydration intravenous fluid bag for cisplatin/carboplatin administration.

Chemotherapy is continued for a maximum of six cycles in the absence of prohibitive toxicity (eg, cumulative neuropathy, hearing impairment, kidney impairment). However, after the patient has completed four cycles, it is up to the judgement of the site investigator whether to complete all six cycles.

Durvalumab is continued if chemotherapy is stopped prior to completion of six cycles in participants with tumours that are stable or responding to treatment.

For participants entering into the maintenance stage, the first dose of durvalumab should commence 3 weeks after the last dose of chemodurvalumab. Subsequent treatments with durvalumab will continue at $1500 \mathrm{mg}$ on day 1 of a 4-weekly cycle in the absence of disease progression, unacceptable toxicity, withdrawal of consent or other reasons for discontinuation.

\section{Control arm (arm B)}

Standard doublet chemotherapy followed by close observation (see table 2 ).

\section{In both arms}

Carboplatin Area Under the Curve, AUC 5 is the initial platinum agent of choice in participants with an estimated $\mathrm{CrCl} 45-59 \mathrm{~mL} / \mathrm{min}$, or those with clinically reported hearing loss. Carboplatin or cisplatin may be chosen for other participants at the discretion of investigators. Participants experiencing unacceptable cisplatin toxicities may be treated subsequently with carboplatin AUC 5 every 3 weeks. Regimens for antiemetic and hydration are as per local institutional guidelines. 
Table 1 Investigational arm treatment

\begin{tabular}{|c|c|c|c|c|c|}
\hline Agent (s) & Dose & Route & Duration & Schedule & Frequency \\
\hline $\begin{array}{l}\text { Cisplatin } \\
\text { or } \\
\text { carboplatin }\end{array}$ & $\begin{array}{l}75 \mathrm{mg} / \mathrm{m}^{2} \\
\text { or } \\
\text { AUC } 5\end{array}$ & Intravenous & $\begin{array}{l}\text { Per institution } \\
\text { practice }\end{array}$ & Day 1 each cycle & $\begin{array}{l}\text { Every } 3 \text { weeks x } 4-6 \\
\text { cycles }\end{array}$ \\
\hline Pemetrexed & $500 \mathrm{mg} / \mathrm{m}^{2}$ & Intravenous & $\begin{array}{l}\text { Per institution } \\
\text { practice }\end{array}$ & Day 1 each cycle & $\begin{array}{l}\text { Every } 3 \text { weeks x 4-6 } \\
\text { cycles }\end{array}$ \\
\hline Durvalumab & $1500 \mathrm{mg}$ & Intravenous & $60 \mathrm{~min}$ & Day 1 each cycle & Every 3 weeks \\
\hline Durvalumab & $1500 \mathrm{mg}$ & Intravenous & $60 \mathrm{~min}$ & Day 1 each cycle & $\begin{array}{l}\text { Every } 4 \text { weeks } \\
\text { Until disease } \\
\text { progression/ } \\
\text { unacceptable toxicity/ } \\
\text { withdrawal of consent }\end{array}$ \\
\hline
\end{tabular}

AUC, Area Under the Curve

Trial oversight and monitoring

DREAM3R is an investigator-initiated, academic trial, conducted as a collaboration between the Thoracic Oncology Group of Australasia, the NHMRC Clinical Trials Centre at the University of Sydney, and PrECOG, a non-profit research company that focuses on cancer clinical trials. The University of Sydney is the sponsor in Australia and New Zealand, PrECOG is the sponsor in USA. This international study will be conducted through a number of regional coordinating centres, each responsible for their own ethics and regulatory approvals, regional monitoring, medical oversight and facilitation of data collection and query resolution. The NHMRC Clinical Trials Centre will be responsible for study coordination, data acquisition, management and statistical analysis. All patients will be given written informed consent prior to study enrolment.

The trial will be monitored by an Independent Safety and Data Monitoring Committee (ISDMC) approximately every 6 months. The ISDMC will advise the Trial Monitoring Committee (TMC) regarding safety, specified matters related to the integrity and potential conclusions of trial data and the appropriateness of continued trial conduct.

The International Trial Steering Committee (ITSC) will oversee study planning, monitoring, progress, review of information from related research and implementation of recommendations from other study committees and external bodies (eg, ethics committees).
The ITSC will consider recommendations from the ISDMC about whether to continue the study as planned, modify, or stop it, based on safety monitoring or other information.

Each regional coordinating centre will constitute its own regional TMC, including a clinical lead and coordinating centre lead who will represent the region on the ITSC.

Changes and amendments to the protocol can only be initiated and made by the ITSC. Approval of protocol amendments by the Institutional Human Research Ethics Committees is required prior to their implementation.

\section{Patient and public involvement}

Patient and members of the public were involved at several stages of the trial, including the design, management and conduct of the trial. We received input from mesothelioma patients in the design of the trial materials and management oversight through membership of the trial steering committee. We carefully assessed the burden of the trial interventions on patients. We intend to disseminate the main results to trial participants and will seek patient and public involvement in the development of an appropriate method of dissemination.

\section{STATISTICAL CONSIDERATIONS \\ Sample size}

Enrolment of 480 participants (randomised 2:1) over 27 months and followed for at least another 24 months

\begin{tabular}{llllll}
\hline \multicolumn{2}{l}{ Table 2} & Control arm treatment & & & \\
\hline Agent (s) & Dose & Route & Duration & Schedule & Frequency \\
\hline $\begin{array}{l}\text { Cisplatin } \\
\text { or } \\
\text { carboplatin }\end{array}$ & $\begin{array}{l}75 \mathrm{mg} / \mathrm{m}^{2} \\
\text { or }\end{array}$ & AUC 5 & Per institution practice & Day 1 each cycle & Every 3 weeks $\times 4$ to 6 cycles \\
$\begin{array}{l}\text { Pemetrexed } \\
\text { Followed by }\end{array}$ & $500 \mathrm{mg} / \mathrm{m}^{2}$ & Intravenous & Per institution practice & Day 1 each cycle & Every 3 weeks $\times 4$ to 6 cycles \\
Close observation per standard of care & & & \\
\hline
\end{tabular}


provides $>85 \%$ power assuming a true HR of 0.70 , a median survival of 15 months in the control group and a median survival of 21.4 months in the durvalumab group. The alternate hypothesis (difference) will be tested against the null hypothesis (no difference) with a wo-sided alpha of 0.05 . There is an allowance for noncompliance with assigned treatment of $6 \%$.

A single interim analysis will be conducted according to the alpha spending approach using an O'Brien-Fleming boundary. The interim analysis will be conducted at least 6 months after the completion of recruitment and having observed $50 \%$ events required for the final analysis. The exact boundary will be computed prior to the analysis according to the percentage of information observed. For example, at exactly 50\% information (176 events), the analysis would use alpha $=0.0031$ and declare a significant result if the observed $\mathrm{HR}<0.64$. The final analysis (352 events) would then be based on with alpha 0.049 and have power of $85 \%$ if the true HR was 0.70 .

\section{Statistical analysis}

All randomised participants will be included in the analysis. With the exception of safety data, all analyses will be conducted on an intention to treat basis (safety analysis will be reported by treatment as received within all participants who received any study treatment). 95\% CIs will be reported for all relevant estimates. A statistical analysis plan will be prepared prior to the final analysis. This document will contain additional detail on the methods described here.

The primary endpoint of the study is OS, defined as the time from randomisation to the date of death due to any cause. Participants who are alive at the time of the final analysis or who have become lost to follow-up will be censored at their last known alive date. All randomised participants will be included in the analysis of OS. KaplanMeier estimates will be computed for both groups. CI for the median survival will be computed by the method of Brookmeyer and Crowley. In the primary analysis, the two treatment arms will be compared using the log-rank test stratified by stratification factors. Cox regression modelling will be used to estimate the treatment effect both on an unadjusted basis and adjusted for stratification variables.

PFS is a secondary endpoint of this study, defined as the time from randomisation to the date of the first documented disease progression (based on mRECIST and iRECIST) or death due to any cause. A patient who stops treatment with study drug and goes onto receive alternative therapy for pleural mesothelioma, prior to documentation of disease progression, will be censored on the date alternative therapy began. If a patient has not progressed or received alternative therapy, PFS will be censored on the date of the last disease assessment. All randomised participants will be included in the analysis of PFS. All analyses for OS will be similarly performed for PFS.

OTR rate is defined as the proportion of participants with a documented complete response, partial response
$(\mathrm{CR}+\mathrm{PR})$ based on iRECIST criteria. Results for tumourrelated endpoints (PFS and ORR) based on iRECIST will be considered exploratory. The primary estimate of OTR rate will be based on all participants randomised, and compared using Cochran-Mantel-Haenszel test stratified by stratification factors. Quality of life analysis will be conducted with appropriate methods to account for repeated measures.

An exploratory analysis of biomarkers (from tissue, serial bloods) and their associations with clinical endpoints will be conducted. These exploratory analyses will be descriptive/graphical in nature and are designed to generate new hypotheses to be tested in future clinical studies. Where parameters of immune response are measured, continuous variables will be summarised with means and SD. Dichotomous and categorical variables will be summarised using proportions with exact $95 \%$ CIs and counts, respectively. These summaries will be computed for each treated patient at multiple time points, before and after treatment administration, as indicated in the study schema. Plots will be used to show the changes in immune response over time for each individual. For each patient, comparisons in the predurvalumab and postdurvalumab responses will be compared using paired $\mathrm{t}$ tests (or Wilcoxon signed rank tests if appropriate) for continuous variables and McNemars test for dichotomous or categorical variables. Associations between immune responses will be explored graphically (eg, scatterplots, boxplots) and numerically (eg, correlations, $\chi 2$ tests).

\section{ETHICS AND DISSEMINATION}

The study will be conducted according to the ICH Guideline for Good Clinical Practice Integrated Addendum to ICH E6 (R1): Guideline for Good Clinical Practice ICH E6(R2) dated 9 November 2016, the principles laid down by the World Medical Association in the Declaration of Helsinki 2013 and pertinent regional regulations.

The study gained central ethical approval for Australia and New Zealand sites from the Sydney Local Health District Ethics Review Committee (RPAH Zone) (2019/ ETH13618) on 17 February 2021 and Northern B Health and Disability Ethics Committee on 26 January 2021. USA sites received initial approval 28 December 2020 from Western Institutional Review Board-WCG IRB Puyallup, Washington.

\section{Trial status}

Patient enrolment commenced in February 2021 at Sir Charles Gardiner Hospital, Perth, Australia. As of 15 September 2021, 41 of the 60 planned sites have opened to recruitment and 45 participants have been randomised.

\section{Author affiliations}

${ }^{1}$ NHMRC Clinical Trials Centre, University of Sydney, Camperdown, New South Wales, Australia

${ }^{2}$ School of Medicine, Western Sydney University, Campbelltown, New South Wales, Australia 
${ }^{3}$ Bloomberg-Kimmel Institute for Cancer Immunotherapy, Johns Hopkins Medicine Sidney Kimmel Comprehensive Cancer Center, Baltimore, Maryland, USA ${ }^{4}$ Medical Oncology, Royal Brisbane and Women's Hospital, Herston, Queensland, Australia

${ }^{5}$ Medicine, The University of Queensland, Saint Lucia, Queensland, Australia ${ }^{6}$ ECOG-ACRIN Biostatistics Center, Frontier Science Foundation, Boston,

Massachusetts, USA

${ }^{7}$ Winship Cancer Institute, Emory University, Atlanta, Georgia, USA

${ }^{8}$ National Centre for Asbestos Related Diseases (NCARD), Institute for Respiratory Health, Perth, Western Australia, Australia

${ }^{9}$ School of Biological Sciences, University of Western Australia, Perth, Western Australia, Australia

${ }^{10}$ Telethon Kids Institute, Nedlands, Western Australia, Australia

${ }^{11}$ Medical Oncology, Princess Alexandra Hospital, Woolloongabba, Queensland, Australia

${ }^{12}$ Queensland University of Technology, Brisbane, Queensland, Australia

${ }^{13}$ Genesis Care, Sydney, New South Wales, Australia

${ }^{14}$ The University of Sydney, Sydney, New South Wales, Australia

${ }^{15}$ PrECOG, Philadelphia, Pennsylvania, USA

${ }^{16}$ Medical School, The University of Western Australia, Perth, Western Australia, Australia

\section{Twitter Peey Sei Kok @peeysei}

Collaborators Authors are members of collaborative groups - Thoracic Oncology Group of Australasia (TOGA), the NHMRC Clinical Trials Centre at the University of Sydney, and PrECOG, USA.

Contributors Conception and design of study: AKN, PMF, MS, SR, JB, NP, CB, ZS BH, PSK. Conduct of study: MMC, KaF, KarF, AB, Acquisition of data: KaF, KarF, AB. Drafting the manuscript: PSK, KaF, KarF, AB Revising the manuscript critically for important intellectual content: AKN, PMF, MS, BH, NP, AC, WJL, SY, MMC. Approval of the version of the manuscript to be published: PSK, PMF, BH, ZS, CB, SR, AC, WJL, SY, KO'B, NP, JB, VA, KaF, KarF, AB, MMC, MS, AKN.

Funding This research was conducted with support from Medimmune Ltd and AstraZeneca Pty Ltd, grant number ESR-19-20283.

Competing interests $\mathrm{ZS}, \mathrm{CB}, \mathrm{AC}, \mathrm{SY}, \mathrm{KaF}, \mathrm{KarF}, \mathrm{AB}, \mathrm{MMC}$ have nothing to disclose. PSK reports grants and fees from AstraZeneca and Pfizer, she is a consultant/ advisory board member of MSD, outside the submitted work; PMF reports grants and fees from AstraZeneca, BMS, Corvus, Novartis, Kyowa; he is a consultant/ advisory board member of Amgen, AstraZeneca, BMS, Novartis, Janssen, Iteos, Mirati, Sanofi; he is a Data Safety Monitoring Board member of Polaris, Flame, outside the submitted work; BH is a consultant/ advisory board member of MSD, BMS, Roche, Pfizer, AstraZeneca, Eisai, Takeda; his institution received grants from Amgen, outside the submitted work; SR reports grants and fees from Amgen, AstraZeneca, Genmab, Eisai, Lilly, Roche, Merck, Takeda and GSK; he is a Data Safety Monitoring Board member of Jansen; a member of Board for Gergia Society of Oncology and IASLC; his institution received grants from AstraZenca, Amgen, BMS, Merck, Genmab, Takeda, Advaxis and Pfizer, outside the submitted work; WJL reports grants and fees from Douglas Pharmaceuticals, and patents relating to immune checkpoint therapy, unrelated to this study, outside the submitted work; $\mathrm{KO}$ 'B has received advisory board and/or speaker bureau and/or meeting travel/ registration support from BMS, MSD, LillyOncology, Boehringer-Ingelheim, Pfizer, Novartis, Roche-Genentech,Teva, Mundipharma, Astrazeneca, Janssen, Natera and Tristar. He is a board member and stock holder for Carpe Vitae Pharmaceuticals and a stock holder for RepLuca Pharmaceuticals and DGC Diagnostics and holds patents for novel therapeutics and diagnostic tests, outside the submitted work; NP reports grants and fees from Boehringer Ingelheim, Bayer, Novartis, Pfizer, Roche, Takeda and Ipsen; he is an advisory board member of Boehringer Ingelheim, MSD, Merck, BMS, Astra Zeneca, Takeda, Pfizer and Roche; his institution received grants from Bayer, Pfizer and Roche, outside the submitted work; JB reports grants and fees from AstraZeneca, BMS, Genentech/Roche, Merck, RAPT Therapeutics, Revolution Medicines, Amgen, Eli Lilly, GlaxoSmithKline, Sanofi, Regeneron; she is a Data Safety Monitoring Board member of GlaxoSmithKline, Sanofi, Janssen; she is an advisory board member of IASLC; outside the submitted work; VA institution received grants from BMS and AstraZeneca; outside the submitted work; MS institution received grants from the following competitive funding bodies: Australian National Health and Medical Research Council, Canadian Cancer Trials Group, Cancer Australia, Medical Research Future Fund of Australia; and the following pharmaceutical companies: Astellas, Amgen, Astra Zeneca, Bayer, Beigene, Bionomics, Bristol-Myers Squibb, Celgene, Medivation, Merck, Merck Sharp \&
Dohme, Pfizer, Roche, Sanofi, Tilray, outside the submitted work; AKN reports grants and fees from Bayer Pharmaceuticals; Roche Pharmaceuticals; Boehringer Ingelheim; Merck Sharpe Dohme; Douglas Pharmaceuticals, Atara Biotherapeutics, Astra Zeneca (payment to institution); Pharmabcine; Trizell Ltd; Seagen; honoraria from Bristol Myers Squibb and her institution received grants from AstraZeneca and Douglas Pharmaceuticals, outside the submitted work.

Patient and public involvement Patients and/or the public were involved in the design, or conduct, or reporting, or dissemination plans of this research. Refer to the Methods section for further details.

Patient consent for publication Not applicable.

Provenance and peer review Not commissioned; externally peer reviewed.

Open access This is an open access article distributed in accordance with the Creative Commons Attribution Non Commercial (CC BY-NC 4.0) license, which permits others to distribute, remix, adapt, build upon this work non-commercially, and license their derivative works on different terms, provided the original work is properly cited, appropriate credit is given, any changes made indicated, and the use is non-commercial. See: http://creativecommons.org/licenses/by-nc/4.0/.

ORCID iD

Peey Sei Kok http://orcid.org/0000-0002-4910-4615

\section{REFERENCES}

1 Robinson BM. Malignant pleural mesothelioma: an epidemiological perspective. Ann Cardiothorac Surg 2012;1:491-6.

2 UK CR. Mesothelioma incidence statistics. Available: https:// www.cancerresearchuk.org/health-professional/cancer-statistics/ statistics-by-cancer-type/mesothelioma/incidence\#headingZero2019

3 Welfare AloHa. Mesothelioma in Australia, 2019. Available: https:// www.aihw.gov.au/reports/cancer/mesothelioma-in-australia-2019/ contents/summary2020

4 Selby K. Mesothelioma incidence and trends. Available: https://www. asbestos.com/mesothelioma/incidence/2021

5 Robinson BWS, Musk AW, Lake RA. Malignant mesothelioma. Lancet 2005;366:397-408.

6 Robinson BWS, Lake RA. Advances in malignant mesothelioma. $N$ Engl J Med 2005;353:1591-603.

7 Nowak AK. Chemotherapy for malignant pleural mesothelioma: a review of current management and a look to the future. Ann Cardiothorac Surg 2012;1:508-15.

8 Vogelzang NJ, Rusthoven JJ, Symanowski J, et al. Phase III study of pemetrexed in combination with cisplatin versus cisplatin alone in patients with malignant pleural mesothelioma. $J$ Clin Oncol 2003;21:2636-44.

9 Zalcman G, Mazieres J, Margery J, et al. Bevacizumab for newly diagnosed pleural mesothelioma in the mesothelioma Avastin cisplatin pemetrexed study (maps): a randomised, controlled, openlabel, phase 3 trial. Lancet 2016;387:1405-14.

10 Grosso F, Steele N, Novello S, et al. Nintedanib plus Pemetrexed/ Cisplatin in patients with malignant pleural mesothelioma: phase II results from the randomized, placebo-controlled LUME-Meso trial. $J$ Clin Oncol 2017;35:3591-600.

11 Alley EW, Lopez J, Santoro A, et al. Clinical safety and activity of pembrolizumab in patients with malignant pleural mesothelioma (KEYNOTE-028): preliminary results from a non-randomised, openlabel, phase 1B trial. Lancet Oncol 2017;18:623-30.

12 Maio M, Scherpereel A, Calabrò L, et al. Tremelimumab as secondline or third-line treatment in relapsed malignant mesothelioma (determine): a multicentre, international, randomised, double-blind, placebo-controlled phase 2B trial. Lancet Oncol 2017;18:1261-73.

13 Disselhorst MJ, Quispel-Janssen J, Lalezari F, et al. Ipilimumab and nivolumab in the treatment of recurrent malignant pleural mesothelioma (initiate): results of a prospective, single-arm, phase 2 trial. Lancet Respir Med 2019;7:260-70.

14 Scherpereel A, Mazieres J, Greillier L, et al. Nivolumab or nivolumab plus ipilimumab in patients with relapsed malignant pleural mesothelioma (IFCT-1501 MAPS2): a multicentre, openlabel, randomised, non-comparative, phase 2 trial. Lancet Oncol 2019;20:239-53.

15 Okada M, Kijima T, Aoe K, et al. Clinical Efficacy and Safety of Nivolumab: Results of a Multicenter, Open-label, Single-arm, Japanese Phase II study in Malignant Pleural Mesothelioma (MERIT). Clin Cancer Res 2019;25:5485-92. 
16 Fennell D, Ottensmeier C, Califano R, et al. PS01.11 nivolumab versus placebo in relapsed malignant mesothelioma: the confirm phase 3 trial. Journal of Thoracic Oncology 2021;16:S62.

17 Popat S, Curioni-Fontecedro A, Polydoropoulou V, et al. A multicentre randomized phase III trial comparing pembrolizumab (P) vs single agent chemotherapy (CT) for advanced pre-treated malignant pleural mesothelioma (MPM): results from the European thoracic oncology platform (ETOP 9-15) PROMISE-meso trial. Annals of Oncology 2019;30:v931.

18 Hassan R, Thomas A, Nemunaitis JJ, et al. Efficacy and safety of avelumab treatment in patients with advanced unresectable mesothelioma: phase 1B results from the javelin solid tumor trial. JAMA Oncol 2019;5:351-7.

19 Calabrò L, Rossi G, Morra A, et al. Tremelimumab plus durvalumab retreatment and 4-year outcomes in patients with mesothelioma: a follow-up of the open label, non-randomised, phase 2 NIBIT-MESO-1 study. Lancet Respir Med 2021;9:969-76.
20 Baas P, Scherpereel A, Nowak AK, et al. First-Line nivolumab plus ipilimumab in unresectable malignant pleural mesothelioma (CheckMate 743): a multicentre, randomised, open-label, phase 3 trial. Lancet 2021;397:375-86.

21 Stewart R, Morrow M, Hammond SA, et al. Identification and characterization of MEDI4736, an antagonistic anti-PD-L1 monoclonal antibody. Cancer Immunol Res 2015;3:1052-62.

22 Nowak AK, Lesterhuis WJ, Kok P-S, et al. Durvalumab with first-line chemotherapy in previously untreated malignant pleural mesothelioma (DREAM): a multicentre, single-arm, phase 2 trial with a safety run-in. Lancet Oncol 2020;21:1213-23.

23 Forde PM, Anagnostou V, Sun Z, et al. Durvalumab with platinumpemetrexed for unresectable pleural mesothelioma: survival, genomic and immunologic analyses from the phase 2 PrE0505 trial. Nat Med 2021;27:1910-20. 